\title{
Aromatase Inhibition Abolishes LTP Generation in Female But Not in Male Mice
}

\author{
Ricardo Vierk, ${ }^{1 \star}$ Günter Glassmeier, ${ }^{2 \star}$ Lepu Zhou, ${ }^{1}$ Nicola Brandt, ${ }^{1}$ Lars Fester, ${ }^{1}$ Danuta Dudzinski, ${ }^{1}$ Wiebke Wilkars, ${ }^{1}$ \\ Roland A. Bender, ${ }^{1}$ Martha Lewerenz, ${ }^{1}$ Simon Gloger, ${ }^{1}$ Lucas Graser, ${ }^{1}$ Jürgen Schwarz, ${ }^{3}$ and Gabriele M. Rune ${ }^{1}$ \\ ${ }^{1}$ Institute of Neuroanatomy, University Medical Center Hamburg-Eppendorf, 20246 Hamburg, Germany, ${ }^{2}$ Institute of Cellular and Integrative Physiology, \\ University Medical Center Hamburg-Eppendorf, 20246 Hamburg, Germany, and ${ }^{3}$ Center of Molecular Neurobiology Hamburg, University Medical Center \\ Hamburg-Eppendorf, 20246 Hamburg, Germany
}

Inhibitors of aromatase, the final enzyme of estradiol synthesis, are suspected of inducing memory deficits in women. In previous experiments, we found hippocampal spine synapse loss in female mice that had been treated with letrozole, a potent aromatase inhibitor. In this study, we therefore focused on the effects of letrozole on long-term potentiation (LTP), which is an electrophysiological parameter of memory and is known to induce spines, and on phosphorylation of cofilin, which stabilizes the spine cytoskeleton and is required for LTP in mice. In acute slices of letrozole-treated female mice with reduced estradiol serum concentrations, impairment of LTP started as early as after $6 \mathrm{~h}$ of treatment and progressed further, together with dephosphorylation of cofilin in the same slices. Theta-burst stimulation failed to induce LTP after 1 week of treatment. Impairment of LTP was followed by spine and spine synapse loss. The effects were confirmed in vitro by using hippocampal slice cultures of female mice. The sequence of effects in response to letrozole were similar in ovariectomized female and male mice, with, however, differences as to the degree of downregulation. Our data strongly suggest that impairment of LTP, followed by loss of mushroom spines and spine synapses in females, may have implications for memory deficits in women treated with letrozole.

\section{Introduction}

The impact of estradiol on hippocampal synaptic plasticity has frequently been shown (for review, see Spencer et al., 2008). It was demonstrated that estradiol induces spine and spine synapse formation in rat hippocampus, a brain region required for learning and memory. Similarly, estradiol treatment increases the magnitude of long-term potentiation (LTP) at hippocampal CA3-CA1 synapses, an electrophysiological parameter of memory. Both phenomena have been related to the memoryenhancing effects of the hormone (Walf et al., 2006).

The effects of estradiol on spine density and LTP were frequently shown by either using ovariectomized animals, which had been treated systemically with estradiol (Gould et al., 1990; Warren et al., 1995; Córdoba Montoya and Carrer, 1997; Smith and McMahon, 2005; Kramár et al., 2009), by applying estradiol to acute slices of mostly male rats (Foy et al., 1999; Ito et al., 1999; Mukai et al., 2007; Kramár et al., 2009), or by using mixed hippocampal slice cultures from neonatal males and females

Received Oct. 21, 2011; revised March 16, 2012; accepted April 10, 2012.

Author contributions: G.M.R. designed research; R.V., G.G., L.Z., N.B., L.F., D.D., W.W., M.L., S.G., and L.G. performed research; R.V., G.G., L.Z., N.B., L.F., D.D., W.W., R.A.B., J.S., and G.M.R. analyzed data; G.M.R. wrote the paper.

This work was supported by the Deutsche Forschungsgemeinschaft (Ru 436/4-1). We thank Brigitte Asmus and Bettina Kruck for their excellent technical assistance.

*R.V. and G.G. contributed equally to this work.

Correspondence should be addressed to Dr. Gabriele M. Rune, Zentrum für Experimentelle Medizin, Institut für

Neuroantomie, Universitätsklinikum Hamburg-Eppendorf, Martinistraße 52, D-20246 Hamburg, Germany. E-mail: rune@uke.uni-hamburg.de.

DOI:10.1523/JNEUROSCI.5319-11.2012

Copyright $\odot 2012$ the authors $\quad 0270-6474 / 12 / 328116-11 \$ 15.00 / 0$
(Murphy and Segal, 1996; Kretz et al., 2004; Mendez et al., 2011). Thus, the vast majority of studies do not consider the potential differences between males and females. Furthermore, the results frequently show that exogenous application of estradiol is primarily effective after having removed estradiol, hence leaving the question open as to whether exogenously applied estradiol actually causes major alterations in preexisting networks (Mendez et al., 2011) or simply rescues the effects in response to estradiol depletion.

We have already demonstrated that hippocampal neurons are capable of synthesizing estradiol de novo (Wehrenberg et al., 2001; Prange-Kiel et al., 2003) and that hippocampus-derived estradiol is required for the maintenance of hippocampal synapses (Kretz et al., 2004). Letrozole, which is a nonsteroidal aromatase inhibitor and is commonly used in the therapy of breast cancer, reliably inhibits estradiol synthesis. Treatment of hippocampal slice cultures and systemic treatment of female mice with letrozole resulted in spine and spine synapse loss and downregulation of synaptic proteins in the hippocampus (Kretz et al., 2004; Rune and Frotscher, 2005; Rune et al., 2006; Zhou et al., 2007).

LTP is accompanied by phosphorylation of cofilin (Fukazawa et al., 2003) and it induces the formation of spines (Yuste and Bonhoeffer, 2001, 2004). Recently, Kramár et al. (2009) demonstrated that estradiol-enhanced LTP in acute slices of male rats is paralleled by phosphorylation of cofilin. The status of phosphorylation of cofilin regulates actin assembly and disassembly. When phosphorylated on Ser3, disassembly of F-actin is inhibited and spines are stabilized (Bramham et al., 2008). If estradiol phos- 
phorylates cofilin and enhances LTP, we hypothesized that inhibition of estradiol synthesis should induce the opposite effects, which in turn may underlie synapse loss in response to aromatase inhibition.

In this study, we show that systemic inhibition of aromatase in mice results in dephosphorylation of cofilin and impairment of LTP, which is followed by loss of mature spines and spine synapses in the hippocampus. The effects were most pronounced in intact females, compared with males and ovariectomized females. Our data suggest that this kind of regulation could underlie memory deficits in women who suffer from breast cancer and who are treated with aromatase inhibitors for therapeutical reasons (Dowsett and Haynes, 2003; Shilling et al., 2005).

\section{Materials and Methods}

\section{Animals}

Wistar rats and C57BL/6 mice (Institute of Neuroanatomy, University Medical Center Hamburg-Eppendorf) were maintained under controlled conditions, and water and food were available ad libitum. Young postnatal rats (4-7 d) were anesthetized and decapitated, and the hippocampi were dissected for slice culture preparation. Embryonic day 18 (E18) rats were used for culturing hippocampal cells in dispersion. Adult female, male, and ovariectomized mice were used for the study of LTP and phosphorylated cofilin ( $p$-cofilin) immunoreactivity in acute hippocampal slices and for spine synapse counts in hippocampi of mice treated with letrozole. All experiments were performed in accordance with the institutional guidelines for animal welfare.

Experiments with animals treated with letrozole

Treatment of mice with letrozole. A group of females $(n=21)$ was deeply anesthetized with an injection of $3.3 \mathrm{ml} / \mathrm{kg}$ of a ketamine-xylazine mixture (ketamine $12 \mathrm{mg} / \mathrm{ml}$, xylazine $0.16 \%$ in saline, i.p.) and ovariectomized. The ovariectomized animals were allowed to recover from ovariectomy for 1 week. Ovariectomized female $(n=21)$, intact female $(n=24)$, and male $(n=21)$ C57BL/6 mice, all 12 weeks of age, were injected intraperitoneally with $10 \mu \mathrm{g}$ per gram of body weight of letrozole in $0.05 \mathrm{ml}$ of alcohol and $0.05 \mathrm{ml}$ of $0.9 \% \mathrm{NaCl}$. Controls were treated with the vehicle. The groups of animals treated for $6 \mathrm{~h}$ and $1 \mathrm{~d}$ were injected once, and the groups of animals treated for 2 and $7 \mathrm{~d}$ were injected with letrozole every morning.

Electron microscopy. For electron microscopic examination, the animals ( $n=12$ females, $n=9$ males; $n=9$ ovariectomized animals) were deeply anesthetized with an injection of the ketamine-xylazine mixture (ketamine $12 \mathrm{mg} / \mathrm{ml}$, xylazine $0.16 \%$ in saline, i.p.) and transcardially perfused with $2.5 \%$ glutaraldehyde in PBS. Blood samples for the measurement of estradiol in serum had been taken shortly before perfusion was started. A commercial estradiol RIA (Diagnostic Systems Laboratories) was used.

Following fixation, tissue samples were postfixed in $1 \% \mathrm{OsO}_{4}$ for 30 min, dehydrated in graded ethanol using $1 \%$ uranyl acetate in $70 \%$ ethanol for $30 \mathrm{~min}$, and then embedded in Epon 820 (Serva). Blocks were trimmed to contain only the stratum pyramidale and radiatum of the CA1 region. Thin sections were cut on a Reichert-Jung OmU3 ultramicrotome. Ultrathin sections were stained with uranyl acetate followed by lead citrate.

The spine synapse density was calculated using unbiased stereological methods as previously described (Prange-Kiel et al., 2004). Briefly, pairs of consecutive serial ultrathin sections were cut and collected on formvar-coated single grids. The sections contained the upper and middle third of the CA1 stratum radiatum. Photographs were taken at a magnification of $6600 \times$ with the observer blinded to the experimental treatment. Areas occupied by interfering structures, such as large dendrites or blood vessels, were intentionally avoided. To obtain a comparable measure of synaptic numbers, unbiased for possible changes in synaptic size, the dissector technique was used (Sterio, 1984). The density of spine synapses of pyramidal cell dendrites was calculated with the help of a reference grid superimposed on the EM prints. The only spine synapses counted were those present on the reference section but not on the look-up section. The dissector volume was calculated by multiplying the unit area of the reference grid by the distance $(0.09 \mu \mathrm{m})$ between the upper faces of the reference and the look-up section (Braendgaard and Gundersen, 1986). At least 10 neuropil fields were photographed on each electron microscopic grid. With at least two grids from each slice, containing at least two pairs of consecutive ultrathin sections, each slice provided a minimum of 20 neuropil fields.

Electrophysiology. For electrophysiological examination, the animals ( $n=12$ females; $n=9$ males; $n=9$ ovariectomized females) were anesthetized with isoflurane and subsequently decapitated. The brains were removed immediately and transferred into ice-cold ACSF. Horizontal hippocampal slices $(300 \mu \mathrm{m})$ were cut by using a microvibratome (VT1000; Leica). Slices were kept for $30 \mathrm{~min}$ at $37^{\circ} \mathrm{C}$ in warm, continuously oxygenized ACSF containing the following (in mM): $125 \mathrm{NaCl}, 25$ $\mathrm{NaHCO}_{3}, 2.5 \mathrm{KCl}, 1.25 \mathrm{NaH}_{2} \mathrm{PO}_{4}, 2 \mathrm{CaCl}_{2}, 1.5 \mathrm{MgCl}_{2}, 22.7$ D-glucose. Slices were either fixed with $4 \%$ paraformaldehyde in PBS for immunohistochemistry (see below), or they were used for electrophysiology and fixed after they had been electrophysiologically measured.

For electrophysiological recordings a Zeiss Axioskop 2FS and micromanipulators (M0-103, Narashige; SM1 + Unit Mini3, Luigs and Neumann) were used. Rat organotypic slice cultures were transferred to a brain slice chamber and continuously perfused with oxygenized ACSF using a peristaltic pump ( $2-3 \mathrm{ml} / \mathrm{min})$. Slices were allowed to equilibrate for at least $30 \mathrm{~min}$ in ACSF before recordings were started at room temperature $\left(22-24^{\circ} \mathrm{C}\right)$.

In all experiments, evoked field EPSPs (fEPSPs) were recorded extracellularly from the stratum radiatum of the CA1 area. Schaffer collaterals were stimulated concomitantly with a second glass electrode placed in the CA3 area, $\sim 250 \mu \mathrm{m}$ from the recording electrode. Both glass electrodes were filled with ACSF and had resistances between 1.0 and $4 \mathrm{M} \Omega$. Stimulation, data acquisition, and analysis were performed using Pulse software in combination with an EPC-10 patch-clamp amplifier (HEKA). The synaptic responses were preamplified (gain of 10) by a Philips PM 5170 amplifier. We used theta-burst stimulation, a pattern consisting of eight bursts applied at $5 \mathrm{~Hz}$, with each burst being composed of four pulses at $100 \mathrm{~Hz}$ and with each pulse lasting $0.2 \mathrm{~ms}$ (modified from Muller et al., 1996, and Bukalo et al., 2004), to induce LTP. At the beginning of each recording, single pulses $(0.2 \mathrm{~ms}$, every $2.5 \mathrm{~s})$ were used to set the stimulus strength, yielding baseline fEPSPs with an amplitude $\leq 50 \%$ from the subthreshold maximum. Synaptic transmission was monitored every $30 \mathrm{~s}$ and the data were digitized at $10 \mathrm{kHz}$. After a 20 min stable baseline recording, theta-burst stimulation was administered to induce LTP in CA1 and was monitored continuously for a further 90 min after high-frequency stimulation. Changes of mean fEPSP slopes (compared with baseline, calculated at $60-70 \mathrm{~min}$ post-tetanus) were used as a measure of LTP.

Data analysis was performed using PulseFit 8.11 (HEKA), SigmaPlot (SPSS), Excel (Microsoft), and SPSS 18.0 (IBM).

Immunohistochemistry and image analysis. Immunohistochemistry was performed as described previously (Kretz et al., 2004). Cryostat sections $(12 \mu \mathrm{m})$ of the hippocampal slice cultures and acute hippocampal slices were incubated overnight at $4^{\circ} \mathrm{C}$ with primary antibodies against MAP-2 (1:500, Millipore) and NR2b NMDAR (1:500, Millipore), and against p-cofilin (1:1000, Santa Cruz Biotechnology). Staining was visualized by the use of the appropriate fluorescence-labeled secondary antibodies (Cy3-labeled, FITC-labeled, and Alexa488-labeled anti-mouse or anti-rabbit antibody, 1:350 or 1:500, Millipore). The nuclei were counterstained with DAPI (1:10,000 in PBS, Sigma-Aldrich).

A confocal laser-scanning microscope (Meta 5, Zeiss) was used for the observation and documentation of cultured slices, and a Zeiss Meta LSM 510 upgrade to ZEN 710 Zeiss software was used to achieve 6-12 threedimensional sections in $\mathrm{CA} 1$ of the stratum radiatum in every acute slice. For quantification and comparison of immunostainings in response to the treatments, sections of slices were photographed immediately after the last step of immunocytochemistry. With nontreated, control sections of the experiments, a threshold of staining was defined as being specific, and all the sections of slices treated in this experiment were subsequently photographed using this threshold. To assay NR2B, NMDAR staining, and $\mathrm{p}$-cofilin immunostaining in $\mathrm{CA} 1$ of the stratum radiatum, areas of a 
defined size were analyzed. A relative staining index (integrated density) was determined by first using a cell imaging system (Openlab 3.1, Improvision) and Image (NIH) to measure stained areas and the intensity of staining and then multiplying the stained area (number of pixels) by the intensity of staining (indicated value on a gray scale) and dividing the result by the number of measured areas. For each group $(n=3)$, the entire stratum radiatum of the CA1 region was analyzed in three independent experiments. To avoid bias, all analyses were performed with the investigator blind to the protocol of the sample under study.

\section{Experiments using hippocampal dispersion cultures}

The effects of letrozole on spine morphology were examined in EGFPexpressing dissociated hippocampal neurons, according to the method of Shi and coworkers (Shi and Ethell, 2006; Shi et al., 2009). Primary hippocampal neurons were transfected with EGFP plasmid using Effectene (Qiagen) according to the manufacturer's instructions to visualize the morphology of the neurons. The neurons were transfected after $13 \mathrm{~d}$ in vitro (DIV) and analyzed $3 \mathrm{~d}$ later at DIV 16. Experimental and control samples were encoded for blind analysis, and GFP-expressing neurons were randomly selected and imaged using a fluorescence microscope (Axiovert2, Zeiss) equipped with a camera (Axiocam HRC, Zeiss). Note that nonspiny neurons were excluded from the analysis. Hidden protrusions that protruded toward the back or front of the viewing plane were not counted. Thirty GFP-expressing neurons were randomly selected from each experimental group and 3-5 proximal dendrites per neuron were analyzed to determine the ratio of thin spines (head diameter, $<0.6$ $\mu \mathrm{m})$ and mushroom spines (head diameter, $>0.6 \mu \mathrm{m}$ ).

\section{Experiments using hippocampal slice cultures}

Slices $(400 \mu \mathrm{m})$ of female hippocampi from newborn rats $(n=18)$ were prepared as described and then cultivated according to the method introduced by Stoppini et al. (1991). In brief, sections were kept at $4^{\circ} \mathrm{C}$ in a preparation solution [Minimal Essential Medium (MEM) and $2 \mathrm{~mm}$ glutamine] during the preparation period for $\sim 10 \mathrm{~min}$. The sections were then placed on moistened translucent membranes $(0.4 \mu \mathrm{m}$ Culture Plate Insert, $30 \mathrm{~mm}$ diameter, Millicell-CM, Millipore), which were inserted in six-well plates ( $35 \mathrm{~mm}$ in diameter) filled with $1 \mathrm{ml}$ of medium $(50 \%$ MEM, 25\% HBSS, 25\% heat-inactivated horse serum) with a final concentration of $2 \mathrm{~mm}$ glutamine and $0.044 \% \mathrm{NaHCO}_{3}$. The $\mathrm{pH}$ was adjusted to 7.3. The cultures were kept in vitro for $11 \mathrm{~d}$ at $37^{\circ} \mathrm{C}$ in a humidified, $\mathrm{CO}_{2}$-enriched atmosphere. The culture medium was changed three times a week. After 4 DIV, the incubation media were supplemented with letrozole $\left(10^{-7} \mathrm{M}\right.$; kindly provided by Novartis) and letrozole plus $17 \beta$-estradiol $\left(10^{-7} \mathrm{M}\right.$, water soluble, Sigma-Aldrich) for $7 \mathrm{~d}$. To test time-dependency, the effect of letrozole was additionally tested for various time intervals. Letrozole did not affect the viability of the cultures, as tested in previous experiments (Fester et al., 2006; Prange-Kiel et al., 2006).

Radioimmunoassay. The medium of nontreated cultures and the medium of cultures treated with letrozole were collected every second day, and the medium from two wells was pooled for estradiol measurement. The processing of the medium and the measurement itself were performed as described previously (Prange-Kiel et al., 2006). In brief, $5 \mathrm{ml}$ of culture supernatant was loaded on a Sep-PAK cartridge (Millipore), which had previously been conditioned with $5 \mathrm{ml}$ of methanol and equilibrated with $5 \mathrm{ml}$ of water. After washing with $5 \mathrm{ml}$ of $0.1 \mathrm{M}$ ammonium acetate buffer, $\mathrm{pH} 4$, to remove hydrophilic compounds, the retained analyte was eluted with $2 \mathrm{ml}$ of methanol. The eluate was dried in vacuo and redissolved in $250 \mu$ l of RIA-assay buffer. Depending on the culture conditions, 50 or $25 \mu \mathrm{l}$ of the sample was analyzed in the estradiol-RIA in duplicates.

Values measured in the unconditioned medium (pure medium, which had not been used for culture) were subtracted as background. Three cultures were measured for each treatment and each dose. To calculate percentage values, the average of the estradiol concentrations determined in the medium collected from the control slice cultures was set at $100 \%$. The values determined in the treatment groups were calculated in relation to the mean value, which was in the range of $700 \mathrm{pg} / \mathrm{ml}$. The mean \pm SEM was calculated. The means were compared using ANOVA followed by a post hoc test (least significant difference). A level of confidence of $p \leq 0.05$ was adopted.

The characteristics of the estradiol-RIA were as follows: crossreactivity of the antiserum $\mathrm{AK} \# 22.2$, generated in our laboratory, was studied by establishing a calibration curve for each steroid tested, and the results were expressed in terms of a percentage of the concentration of the reacting compound at $50 \%$ binding of the $0 \mathrm{pg} / \mathrm{ml}$ estradiol standard. Among the variety of steroids tested, only oestriol (2\%) and oestrone $(10 \%)$ exhibited detectable cross-reactivity. The analytical sensitivity, calculated from the mean, minus two SDs of 20 replicates of the $0 \mathrm{pg} / \mathrm{ml}$ estradiol standard measured in the same run, was $2.1 \mathrm{pg} / \mathrm{ml}$. The intraassay precision was determined from the mean of eight replicates, each with three samples of culture medium spike with 5, 50, and $500 \mathrm{pg} / \mathrm{ml}$ estradiol measured in the same assay. The intraassay coefficients of variation (CVs) were $10.6 \%, 5.9 \%$, and $6.2 \%$, respectively. The interassay precision was determined from the mean of these three spiked medium samples run in duplicate, each in eight consecutive assays. The interassay CVs were $12.1 \%, 6.3 \%$, and $7.1 \%$, respectively.

Western blot. Hippocampal cultures from a subgroup of rat pups $(n=$ 12) were used for Western blot analysis. Hippocampal slices from each rat pup were divided equally into two experimental groups (resulting in $6-8$ cultures per group), which were subjected either to treatment with letrozole $\left(10^{-7} \mathrm{M}\right)$ or were left untreated as controls for $7 \mathrm{~d}$. At the end of the culture period, slices of each experimental group (separately for each pup) were pooled and fast-frozen on dry ice. Deep-frozen cultures were later thawed at $4^{\circ} \mathrm{C}$, then manually homogenized $(15 \% \mathrm{w} / \mathrm{v})$ with a buffer containing $50 \mathrm{~mm}$ Tris- $\mathrm{HCl}, \mathrm{pH}$ 7.4,150 mm NaCl, 1\% Nonidet P-40, $0.1 \%$ SDS, $0.5 \%$ sodium deoxycholate, $5 \mathrm{~mm}$ EDTA, and a mixture of proteinase inhibitors. The homogenates were centrifuged at 20,000 $\times g$ at $4^{\circ} \mathrm{C}$ for $20 \mathrm{~min}$, and the supernatant was collected and frozen until further use. For analysis, extracts were run on $12 \%$ SDS-PAGE. Samples were boiled for $10 \mathrm{~min}$, briefly cooled on ice, and then separated at a voltage that prevented excessive heat. Proteins were blotted on nitrocellulose membranes. The blots were treated with $5 \%$ nonfat milk powder solution (in PBS plus $0.3 \%$ Triton X-100) and incubated with antibodies against $\mathrm{n}$-cofilin or p-cofilin (both polyclonal, derived from rabbit; 1:250; Santa Cruz Biotechnology) for $12 \mathrm{~h}$ at $4^{\circ} \mathrm{C}$. Antibody binding was detected using the ECL-Plus kit (GE Healthcare) and quantified by densitometry using ImageJ software (Abramoff et al., 2004). Glycerinaldehyde-3-phosphate-dehydrogenase (GAPDH) expression, which was not affected by either treatment, was determined as an internal standard (using monoclonal mouse-antiGADPH from Ambion; 1:10.000).

Electrophysiology. Electrophysiology was performed as described above. To show the participation of NMDARs and AMPARs in LTP maintenance in hippocampal slice cultures, $10 \mu \mathrm{M} \mathrm{D}-(-)$-2-amino-5phosphonovalerate (APV) or 6-cyano-7-nitroquinoxaline-2,3-dione (CNQX) were applied after 90 min of stable LTP recording.

Electron microscopy. Electron microscopy and spine synapse counts were performed as described above.

\section{Statistics}

All data from spine counts and from quantification of p-cofilin immunoreactivity using image analysis, in the text as well as in the figures, are presented as means \pm SEM. For p-cofilin immunoreactivity, means \pm SEM were compared using ANOVA followed by a post hoc (Tukey's) test. A level of confidence of $p \leq 0.05$ was adopted. Western blot data were statistically analyzed using paired $t$ tests (experimentally treated cultures were always compared with the respective controls from the same rat; see above), and presented as "expression relative to GAPDH" (mean \pm SEM; $n=1$ refers to pooled slices deriving from one rat pup). Slopes of fEPSPs are given as means \pm SEM. throughout. To compare mean fEPSP slopes between nontreated and letrozole-treated slice cultures, and between vehicle-treated and letrozole-treated acute slices, the paired $t$ test was also used and presented as percentage changes of fEPSP slopes (compared with the baseline). 


\section{Results}

In previous reports, we showed that hippocampal neurons are capable of synthesizing estradiol de novo (Prange-Kiel et al., 2003; Fester et al., 2006). Hippocampal estradiol synthesis is reliably suppressed by aromatase inhibitors, such as letrozole. The expression of estrogen receptors and synaptic proteins, and in particular spine synapse density in the hippocampus, correlated positively with aromatase activity in females (Prange-Kiel et al., 2003, 2006; Kretz et al., 2004; Zhou et al., 2010). All effects following treatment with letrozole were rescued by additional application of estradiol (Kretz et al., 2004; Zhou et al., 2007).

In this study, we injected mice systemically with letrozole over different time periods to unravel the underlying mechanisms of spine synapse loss in response to aromatase inhibition. From previous findings by Yuste and Bonhoeffer (2001) and Fiala et al. (2002), showing that LTP induces spine formation, we speculated that loss of spines and spine synapses after aromatase inhibition may result from LTP impairment. In addition, dendritic spines largely depend on an intact actin cytoskeleton (Matus, 2000), and hippocampal LTP is accompanied by enhanced F-actin assembly within dendritic spines. The assembly of F-actin requires phosphorylation of cofilin. Since the $\mathrm{n}$-cofilin/p-cofilin ratio is indicative of F-actin assembly and the stability of spines (Fukazawa et al., 2003), we studied LTP and p-cofilin immunoreactivity in acute slices of letrozole-treated mice. To elucidate the significance of ovarian estradiol, we compared intact female mice with ovariectomized females. Moreover, as it is impossible to remove all sources of estradiol (i.e., fat tissue, adrenals, testes, and ovaries) without affecting the animals' survival, we also used hippocampal slice cultures of females and treated them with letrozole. We also studied the effects of systemic letrozole treatment in males to take into account potential differences between genders. As a second approach, we compared spine density and the frequency of spine types in single male and female dissociated EGFP-transfected neurons.

As previously shown, estradiol could not be detected in the serum of the animals after systemic treatment of mice with letrozole at a dose of $10 \mu \mathrm{g} / \mathrm{g}$ body weight (Zhou et al., 2010). The measurement of estradiol in the collected media of hippocampal slice cultures revealed considerable amounts of 17 $\beta$-estradiol, as previously shown (Kretz et al., 2004). In this study, we showed that the dose of $10^{-7} \mathrm{M}$ results in maximal inhibition of estrogen synthesis in slice cultures. Supplementation of the medium with letrozole $\left(10^{-7} \mathrm{M}\right)$ led to a significant downregulation of estradiol release into the supernatant (control, $24.2 \mathrm{pg} / \mathrm{ml}$ medium; letrozole, $7.8 \mathrm{pg} / \mathrm{ml}$ medium; $n=3, p \leq 0.05$ ).

\section{Aromatase inhibition impairs LTP}

LTP was studied in acute slices of animals treated with letrozole and compared with acute slices of animals that had received only the vehicle. The vehicle had no effect, as no difference was seen between untreated and vehicle-treated animals (data not shown). In addition, reliable effects were not seen when we applied letrozole directly to acute slices for 3-4 h after stable recording of LTPs for $90 \mathrm{~min}$. In acute slices of untreated and vehicle-treated male, female, and ovariectomized animals, theta-burst stimulation induces LTP, with no significant differences in the magnitude between the groups of animals (Fig. 1). In acute slices from letrozole-treated animals, a reduction in the magnitude of LTP was found (Figs. 2, 3). This was true for all animals: letrozoletreated male mice, letrozole-treated female mice, and letrozoletreated ovariectomized animals.

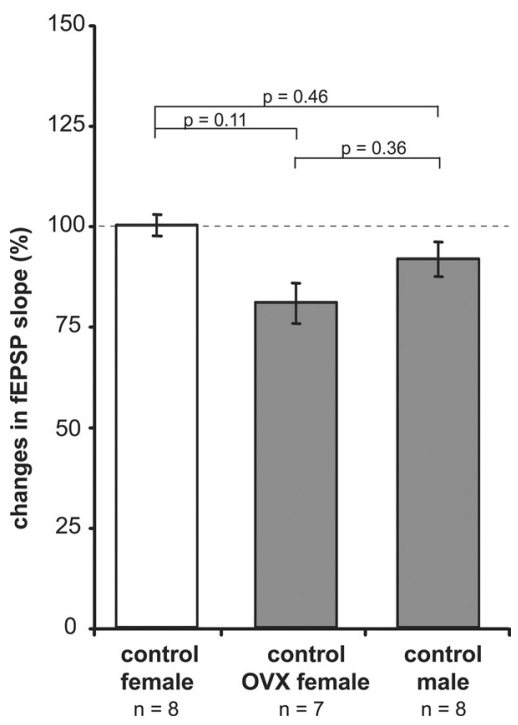

Figure 1. Theta-burst stimulation-induced LTP in acute slices of untreated females, ovariectomized females, and males. The magnitude of the fEPSP slopes were similar in all groups. OVX, ovariectomized; $n=$ number of acute slices of three animals; mean \pm SEM.

In acute slices of vehicle-treated females, theta-burst stimulation of the Schaffer collaterals resulted in LTP. Mean fEPSP slopes were significantly increased to $247 \pm 9 \%$ (compared with the baseline; see Fig. 12A). No difference was found at the various time points in the group of vehicle-treated animals (Fig. $2 A-C$ ). In contrast, a significant reduction of LTP by $\sim 50 \%$ was found as early as after $6 \mathrm{~h}$ of letrozole treatment in female mice (Fig. $2 A, D)$. The LTP impairment in response to letrozole progressed further with an additional 10\% impairment found after $1 \mathrm{~d}$ (Fig. $2 B, D)$. Theta-burst stimulation failed to induce LTP after a treatment lasting $7 \mathrm{~d}$ (Fig. 2C,D).

In male mice, LTP was far less impaired after letrozole treatment than in females. The reduction of LTP was $20 \%$ after $1 \mathrm{~d}$ and remained on this level for up to $7 \mathrm{~d}$ of treatment (Fig. 3A).

In ovariectomized animals, LTP was reduced by $50 \%$ after both 1 and $7 \mathrm{~d}$ of treatment (Fig. 3B). Notably, LTP was not impaired after ovariectomy alone, although ovariectomy substantially reduces estradiol concentrations in serum (Fester et al., 2011). Thus, estradiol of ovarian origin does not seem to play a role in hippocampal LTP. Surprisingly, LTP is less impaired after letrozole treatment of ovariectomized animals compared with intact females (compare Figs. 3B, 2D). Thus, ovariectomy and concomitant decreased levels of estradiol in serum appear to be protective against LTP impairment after letrozole treatment.

The effect of hippocampus-derived estradiol on LTP was further investigated by using hippocampal slice cultures of female animals as a second approach. This in vitro system, besides excluding all influences of nonhippocampal sources of estradiol, preserves synaptic connectivity. Furthermore, the system is well established for determining the density of synapses (Kretz et al., 2004) and for studying LTP under experimental conditions (Mellentin et al., 2006). After $4 \mathrm{~d}$ of preculture, we treated the slice cultures for 1 week with letrozole at a dose of $10^{-7} \mathrm{M}$. We measured theta-burst stimulation-induced LTP at Schaffer collaterals after $7 \mathrm{~d}$ of treatment and compared nontreated hippocampal slice cultures with slice cultures treated with letrozole and slice cultures treated with letrozole plus estradiol.

We first addressed putative negative effects of long-lasting letrozole-treatment on the viability and healthiness of hippocam- 
A
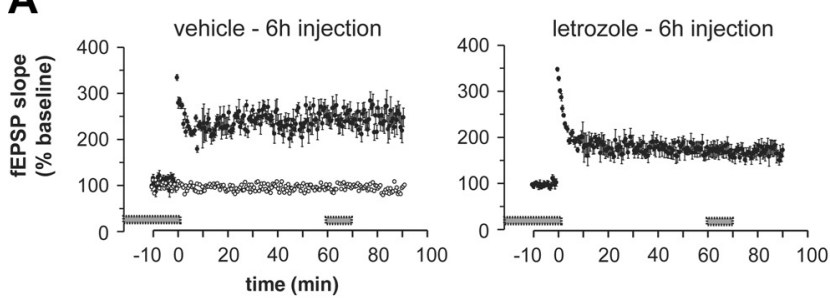

B
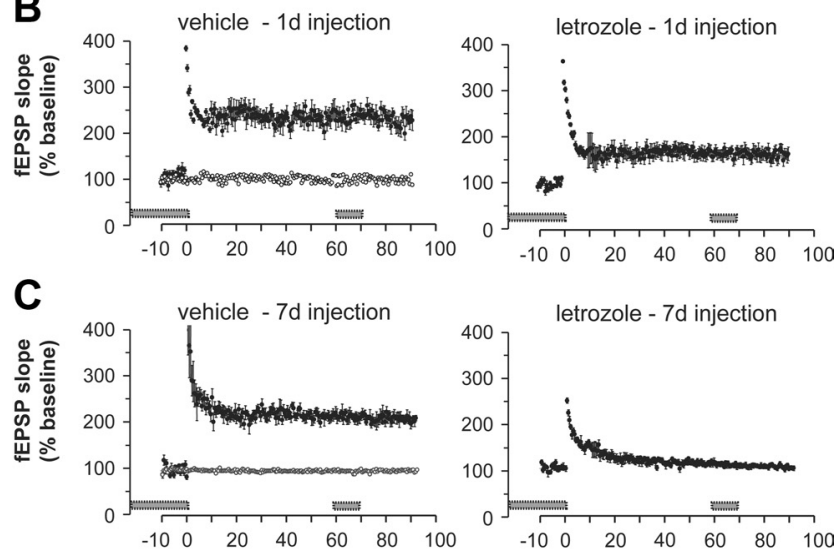

D

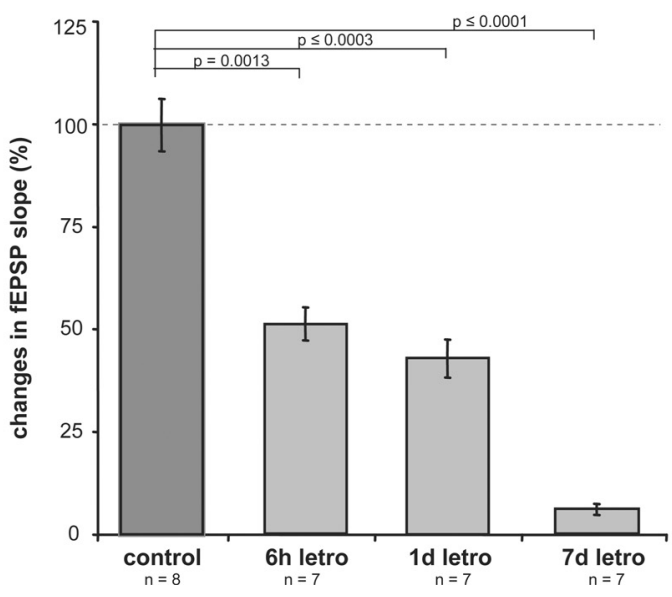

Figure 2. LTP in acute hippocampal slices of letrozole-treated female mice. Results of vehicle-treated animals and letrozole-treated animals. $A$, In slices of vehicle-treated animals ( 6 h), the average course of the fEPSP slopes had significantly increased after 60 min to $247 \pm 9 \%$ $(n=8)$, whereas in letrozole-treated animals the slopes of fEPSPs increased only to $175 \pm 4 \%$ $(n=7) . \boldsymbol{B}$, After $1 \mathrm{~d}$ of vehicle treatment, the slopes of fEPSPs increased to $226 \pm 8 \%(n=8)$ and, after letrozole-treatment for $1 \mathrm{~d}$, the fEPSP slopes had changed only to $154 \pm 5 \%(n=7)$. C, After $7 \mathrm{~d}$ of vehicle injection, the average slope of the fEPSPs increased to $213 \pm 3 \%(n=6)$; in contrast, letrozole injections after $7 \mathrm{~d}$ resulted in virtually unchanged fEPSP slopes, $107 \pm 2 \%$ $(n=7)$. Mean \pm SEM; time point 0 min represents theta-burst stimulation; fEPSP slopes before and $60-70 \mathrm{~min}$ after theta-burst stimulation were quantified and are indicated by gray bars. $\boldsymbol{D}$, Quantitative examination of changes in the average course of the fEPSP after letrozole treatment for $6 \mathrm{~h}$ and for 1 and $7 \mathrm{~d}$ illustrated a highly significant reduction in LTP by $49 \pm$ $4 \%, 57 \pm 5 \%$, and $94 \pm 1 \%$ respectively in females. $n=$ number of acute slices of 3 animals; mean \pm SEM.

pal slice cultures in control experiments. We recorded spontaneous activity of CA1 hippocampal neurons before and after $7 \mathrm{~d}$ of treatment. Figure 4 shows that excitability of these neurons, like spontaneous activity, is similar to the activity of neurons after $7 \mathrm{~d}$ of letrozole-treatment. Basic intrinsic properties, such as resting potential and input resistance (see Fig. 4, legend) were unchanged and in the normal range, as described previously by Podlogar and Dietrich (2006) in response to letrozole. Finally, to
A

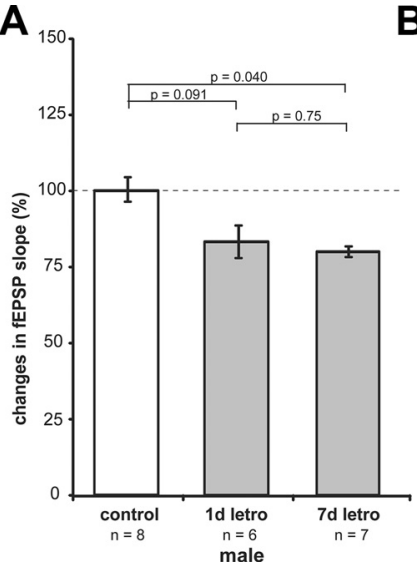

B

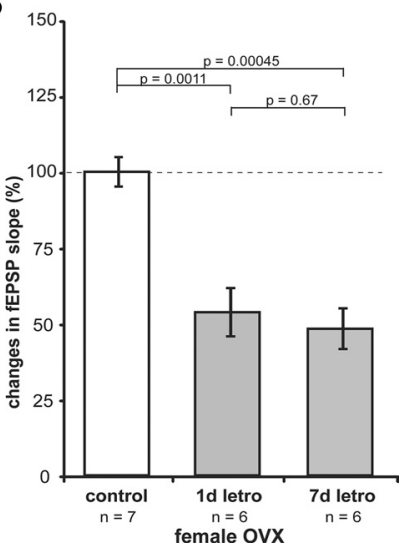

Figure 3. Quantitative examination of changes in the average course of fEPSP slopes after letrozole treatment in ovariectomized females and in males. $A$, Letrozole-treatment of male animals resulted in reduced LTP by $17 \pm 5 \%$ after $1 \mathrm{~d}$ and $20 \pm 2 \%$ after $7 \mathrm{~d}$ treatment. OVX, ovariectomized; mean \pm SEM. $\boldsymbol{B}$, In slices of ovariectomized female mice, LTP was reduced by $46 \pm 8 \%$ after $1 \mathrm{~d}$ of letrozole treatment and $52 \pm 7 \%$ after $7 \mathrm{~d}$ treatment, compared with nontreated ovariectomized animals. $n=$ number of acute slices of three animals; mean \pm SEM.

A

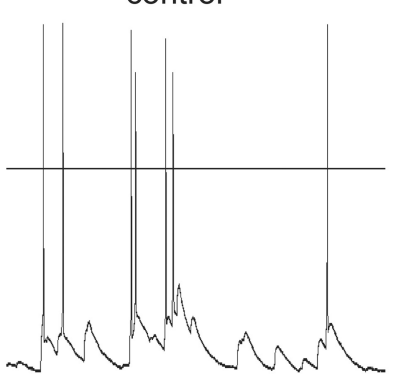

B

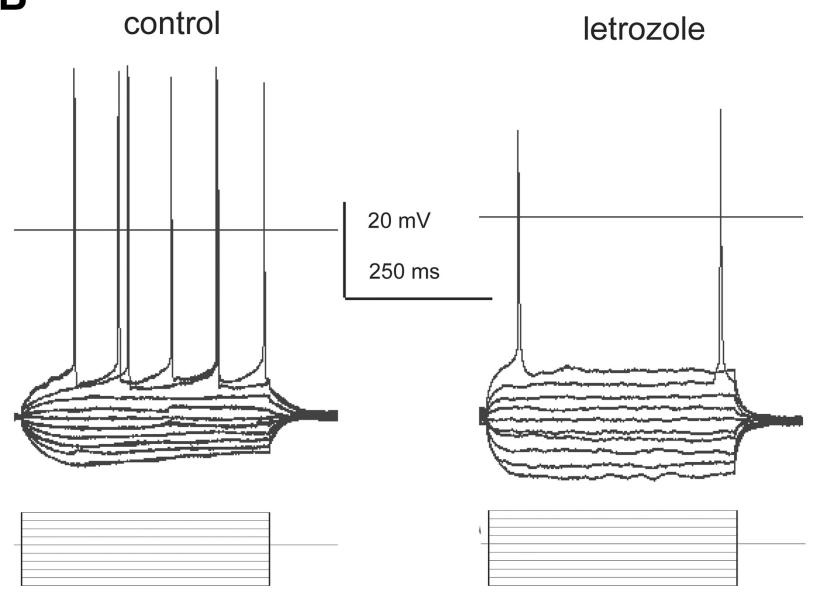

Figure 4. Viability of hippocampal slice cultures in response to letrozole treatment. $\boldsymbol{A}$, Current-clamp recordings of spontaneous activity of hippocampal CA1 neurons under control conditions and after letrozole treatment for $7 \mathrm{~d}$. In both cases, the neurons generated action potentials and received synaptic inputs. $\boldsymbol{B}$, Current-clamp recordings of a hippocampal CA1 neuron under control conditions and after letrozole treatment for $7 \mathrm{~d}$. Neurons were depolarized and hyperpolarized by current pulses from $-50 \mathrm{pA}$ to $+40 \mathrm{pA}$ in steps of $10 \mathrm{pA}$ in the control and from $-100 \mathrm{pA}$ to $+80 \mathrm{pA}$ in steps of $20 \mathrm{pA}$ in letrozole cultures (see pulse diagram). Between control neurons and neurons treated with letrozole, no significant differences were detected between resting potential (controls: $-64.6 \pm 3.9 \mathrm{mV}, n=8$; letrozole: $-66.7 \pm$ $7.6 \mathrm{mV}, n=7$ ) and input resistance (controls: $303 \pm 98 \mathrm{M} \Omega, n=8$ slice cultures of 3 animals; letrozole: $202 \pm 91 \mathrm{M} \Omega$ ) 


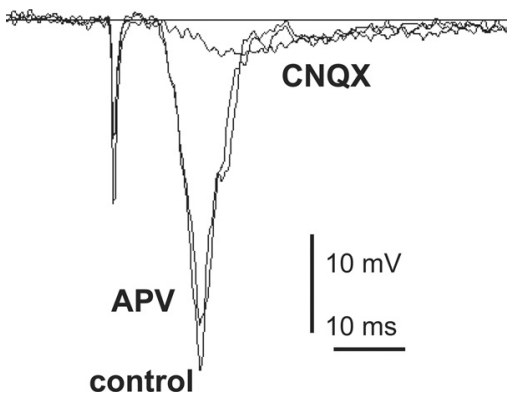

Figure 5. Effect of ionotropic glutamate receptor blocker APV and CNQX on extracellularly recorded field potential (fEPSP) in hippocampal slice cultures. Representative fEPSP traces recorded under normal conditions and in the presence of NMDAR blocker (APV) or AMPAR blocker (CNQX) 90 min after theta-burst stimulation. Like in acute slices, NMDARs contribute less than AMPARs to currents underlying fEPSP (APV: $-19.5 \pm 2.8 \%, n=9, p \leq 0.001$; CNQX: $-83.7 \pm 14.1 \%, n=9 ; p \leq 0.0001) . n=5$ slice cultures of three animals.

A

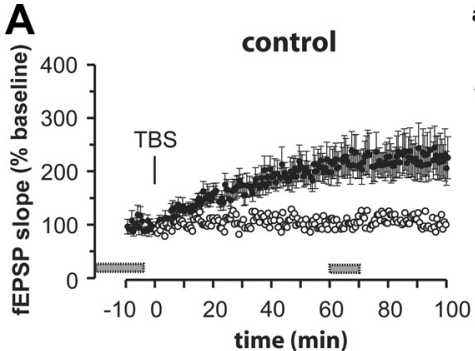

B
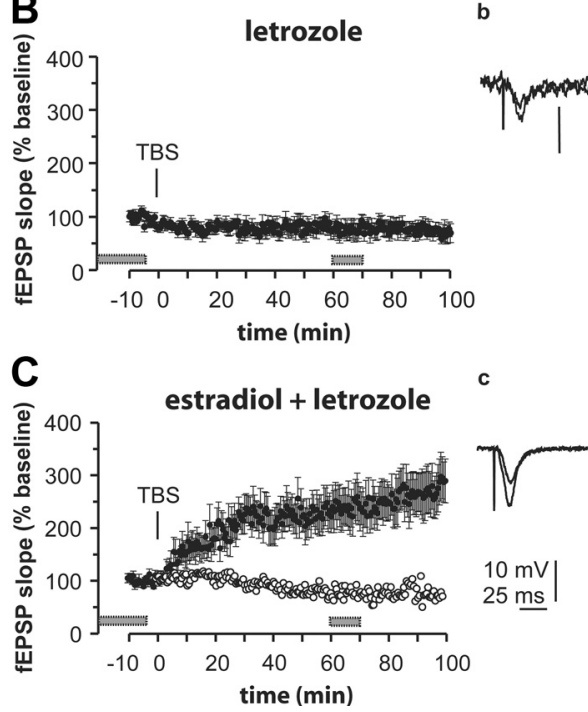

Figure 6. LTP in hippocampal slice cultures of female animals. Theta-burst stimulation was performed after $20 \mathrm{~min}$ of stable baseline recording (10 min are shown) and 60-70 min after theta-burst stimulation (as indicated by gray bars) changes in mean fEPSP slopes were determined. $\boldsymbol{A}$, In untreated slices, the average time course of the fEPSP slopes had significantly increased after 60 min up to $215 \pm 34 \%$. B. Theta-burst stimulation did not induce LTP in slice cultures treated with letrozole for $7 \mathrm{~d}$. C, LTP is rescued by estradiol in letrozole-treated slice cultures. After $60 \mathrm{~min}$, the time course of the fEPSP slope had significantly increased to $239 \pm$ $35 \%$ (mean \pm SEM; $n=6$ slice cultures of 3 animals per group; a, time course of LTP; $b$, superimposed recordings of fEPSPs $10 \mathrm{~min}$ before and $60 \mathrm{~min}$ after theta-burst stimulation).

find out whether the maintenance of theta-burst stimulationinduced LTP in hippocampal slice cultures is NMDAR-dependent and similar to LTP in acute slices, we tested the receptor antagonist APV for NMDARs and CNQX for AMPARs. As demonstrated in Figure 5, NMDARs, in contrast to AMPARs, contribute little to currents underlying a fEPSP in hippocampal slice

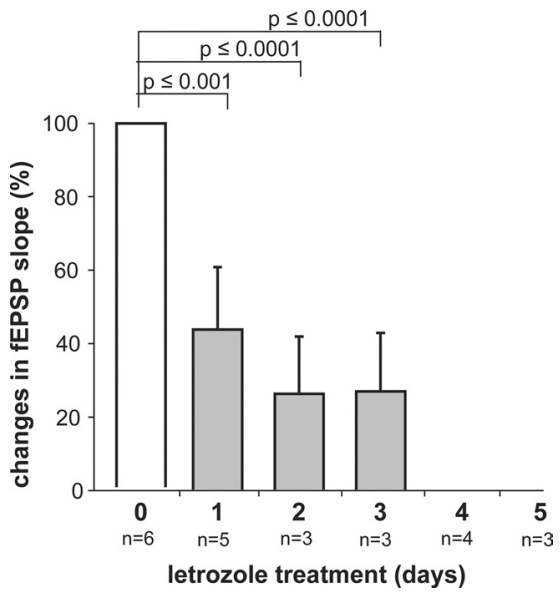

Figure 7. Time course of LTP impairment after aromatase inhibition in female hippocampal slice cultures. Treatment with letrozole for $1 \mathrm{~d}$ resulted in a significant reduction $(53 \pm 20 \%)$ of the mean fEPSP slopes. Longer lasting treatment with letrozole further decreased the course of the fEPSP significantly and, after $4 d$ of treatment, the mean fEPSP course remains unchanged $60 \mathrm{~min}$ after high-frequency stimulation $(n=$ number of slice cultures of 3 animals; means \pm SEM).

cultures. This was also demonstrated in acute slices (Malenka and Nicoll, 1999; Lynch, 2004).

Since recent findings have demonstrated that estradiolinduced LTP is mediated by the NR2b NMDAR (Smith and McMahon, 2006; Snyder et al., 2011), we studied the immunoreactivity in the CA1 region of our hippocampal slices cultures following treatment with letrozole for further control purposes. Consistently, immunoreactivity of the NR2b NMDAR was reduced by $52 \%$ in the stratum radiatum of CA1, as observed by confocal laser scanning microscopy and subsequent digital image analysis ( $n=3$ independent cultures; $p \leq 0.01$, data not shown).

Figure $6 A a-C c$ shows the average time courses of the fEPSP slopes in nontreated, letrozole-treated, and letrozole-plusestradiol-treated slice cultures. After $20 \mathrm{~min}$ of stable baseline recording, theta-burst stimulation of the Schaffer collaterals resulted in a gradual buildup of LTP over $45 \mathrm{~min}$ and did not decay within $100 \mathrm{~min}$ after theta-burst stimulation (Fig. 6A). Similar time courses of LTP induction in organotypic hippocampal slices have been reported previously (Behnisch et al., 2004; Mellentin et al., 2006). Mean fEPSP slopes were measured 60-70 min after theta-burst stimulation and were significantly increased to $215 \pm$ $34 \%$ in nontreated slice cultures. In slice cultures treated for $7 \mathrm{~d}$ with letrozole, however, theta-burst stimulation failed to induce LTP (Fig. 6B), indicating that aromatase activity is obviously required for theta-burst stimulation-induced LTP. To test the specificity of the letrozole effects, we also treated the hippocampal slice cultures with letrozole together with estradiol. Similar to the rescue of letrozole-induced spine synapse loss by estradiol, which we had previously shown (Zhou et al., 2007; Fester et al., 2009), the induction of LTP was preserved at the control level when estradiol and letrozole were simultaneously added to the slice cultures (Fig. 6C). Since theta-burst stimulation failed to induce LTP after $7 \mathrm{~d}$ of letrozole treatment, we studied the time dependency of letrozole effects. As shown in Figure 7, LTP was impaired by $60 \%$ after $1 \mathrm{~d}$. The impairment progressed further and had reached its maximum after $4 \mathrm{~d}$ of treatment.

Impairment of LTP is accompanied by transient dephosphorylation of cofilin in males and females In the acute slices of male, female, and ovariectomized letrozoletreated mice, which we had used for the study of LTP, we also 
quantified immunohistochemistry of p-cofilin in the stratum radiatum of CA1. In control experiments, we found that the staining intensity of p-cofilin was similar in slices immediately fixed after sectioning and was comparable to the intensity in slices that had been processed but were not used for the recordings and were fixed thereafter (data not shown). Immunoreactivity of p-cofilin was increased after theta-burst stimulation-induced LTP in all groups (Fig. 8). This finding is in agreement with Fukazawa et al. (2003), who demonstrated this effect in acute slices of rats.

In the acute slices of letrozole-treated intact female mice, quantification of p-cofilin immunoreactivity revealed a downregulation after $6 \mathrm{~h}$ and after $1 \mathrm{~d}$ of treatment, which highly corresponded to the effects on LTP. p-Cofilin was upregulated after $7 \mathrm{~d}$ of letrozole treatment, which may indicate the onset of compensatory mechanisms (Fig. 9A). Note that this upregulation of p-cofilin occurs despite maximal reduction of LTP at this time point (Fig. 2C,D). The transient character of downregulation was also seen in males (Fig. 9B). Notably, the same degree of cofilin dephosphorylation in males and females resulted in different levels of LTP impairment after $1 \mathrm{~d}$; $20 \%$ in males versus $60 \%$ in females. In ovariectomized animals, p-cofilin immunoreactivity was less reduced compared with that in intact females after $1 \mathrm{~d}$ and remained at this level up to $7 \mathrm{~d}$ in response to letrozole treatment (Fig. 9C). This is very similar to the time course of LTP in ovariectomized animals (Fig. 3C). Western blot analysis of cofilin in female hippocampal slice cultures showed a decrease in p-cofilin and an increase in n-cofilin (Fig. 10), which is consistent with the results of LTP impairment in hippocampal slice cultures (Fig. 6).

\section{LTP impairment precedes spine synapse loss}

Stereological counts of spine synapses, as evidenced by presynaptic bouton, postsynaptic density, and postsynaptic spine observations, revealed spine synapse loss already after $1 \mathrm{~d}$, in response to aromatase inhibition in females (Fig. 11A). The reduction in the number of spine synapses, however, was not significant until $2 \mathrm{~d}$ of treatment of the animals. After $7 \mathrm{~d}$, a $26 \%$ reduction in spine density was observed in female mice (Fig. 11A). After treatment with letrozole for 2 and $7 \mathrm{~d}$, a spine synapse reduction by $10 \%$ was also seen in ovariectomized females compared with the ovarectomized controls, and by $30 \%$ compared with intact female mice (Fig. $11 \mathrm{~B})$. Ovariectomy alone induced a decrease in spine synapse density by $20 \%$. A decrease in the number of spine synapses by $\sim 38 \%$ was found in the stratum radiatum of CA1 of hippocampal slice cultures after $7 \mathrm{~d}$ of treatment (control $7.4 \pm 0.4$ and letrozole $4.5 \pm 0.2$ spine synapses/ 6.4 $\mu \mathrm{m}^{3}, n=3$ independent cultures; $p \leq$ $0.001)$.

Spine synapse loss in response to letrozole was not found in males, de-

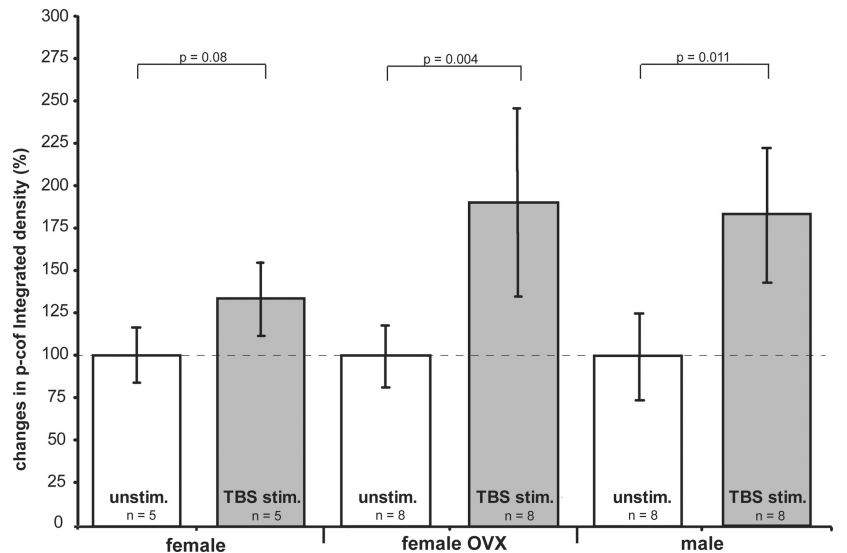

Figure 8. Theta-burst stimulation-induced LTP is accompanied by phosphorylation of cofilin. An increase in p-cofilin immunoreactivity after theta-burst stimulation was observed in acute hippocampal slices of all groups under control conditions: females, ovariectomized females, and males. OVX, ovariectomized; $n=$ number of acute slices of three animals; mean \pm SEM.
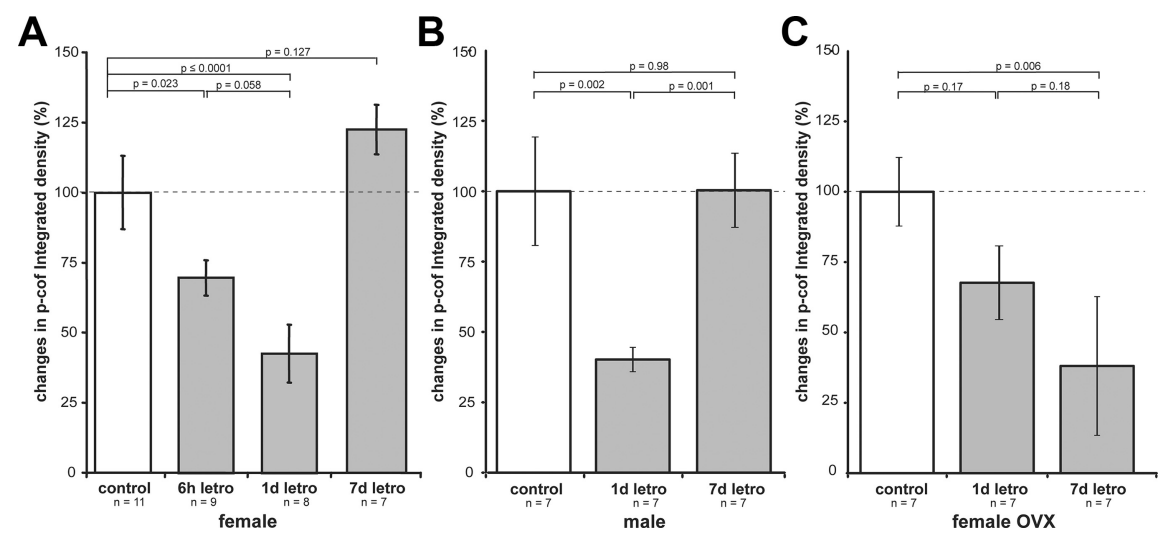

Figure 9. p-Cofilin in acute hippocampal slices of letrozole-treated mice after theta-burst stimulation. Quantitative evaluation of $p$-cofilin immunoreactivity in the stratum radiatum of the CA1 area of slices that had been used for the study of LTP. $A$, Slices of mice treated with letrozole for $6 \mathrm{~h}$ showed a significant reduction in $\mathrm{p}$-cofilin stainings by $30 \pm 6 \%$, whereas longer treatment (1 d) resulted in significantly decreased p-cofilin stainings by $57 \pm 10 \%$. In contrast, after $7 \mathrm{~d}$, p-cofilin is dramatically increased. $\boldsymbol{B}$, $1 \mathrm{~d}$ letrozole treatment resulted in significant reduction of $\mathrm{p}$-cofilin immunoreactivity by $59 \pm 4 \%$ in male animals, but remained unchanged after $7 \mathrm{~d}$ treatment $(0 \pm 13 \%)$. C, Slices from ovariectomized animals treated with letrozole for $1 \mathrm{~d}$ showed a significant reduction in $\mathrm{p}$-cofilin stainings by $32 \pm 13 \%$. This reduction further progressed up to $7 \mathrm{~d}$ of treatment. A significant reduction by $62 \pm 24 \%$ was observed after $7 \mathrm{~d}$. OVX, ovariectomized; $n=$ number of acute slices of three animals; mean \pm SEM.

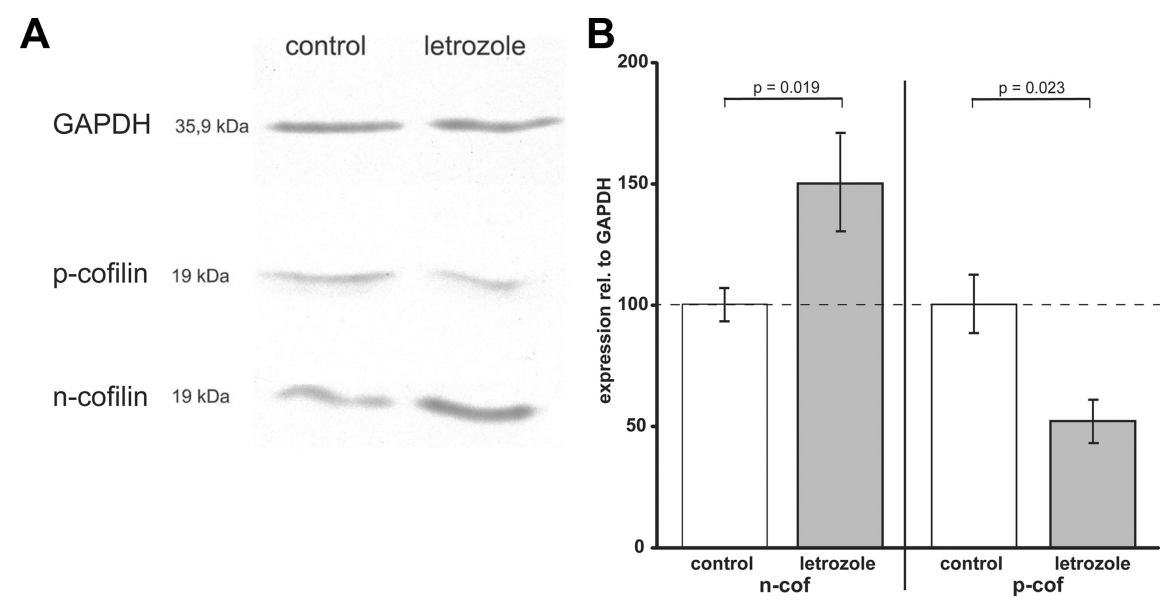

Figure 10. Changes in cofilin expression after letrozole treatment in female hippocampal slice cultures. $A$, Western blot analysis of cofilin after letrozole treatment. $\boldsymbol{B}$, Quantitative evaluation reveals that letrozole significantly downregulates $p$-cofilin and consistently upregulates $n$-cofilin (means $\pm S E M ; n=12$ with 2 Western blots each). 
A
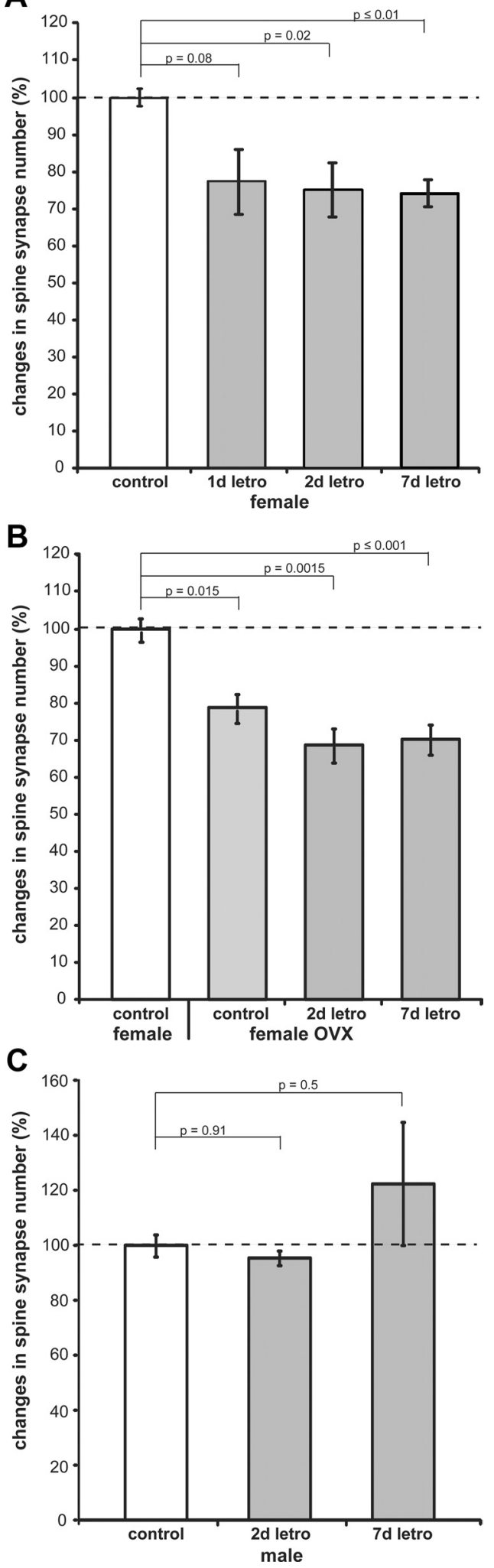

Figure 11. Spine synapse number in mice treated with letrozole. $\boldsymbol{A}$, Significant spine synapse loss was not seen before $2 \mathrm{~d}$ of treatment had elapsed. $\boldsymbol{B}$, In ovariectomized (ovx) letrozoletreated animals, spine synapse number was reduced by $10 \%$ compared with ovariectomized controls and by $30 \%$ compared with untreated intact females. C, No spine synapse loss was seen in males ( $n=3$ animals per group; mean \pm SEM). spite impaired LTP by $20 \%$. This clear-cut difference between males and females prompted us to study spine density and spine phenotype in single dissociated neurons of male and female animals after $2 \mathrm{~d}$ of letrozole exposure.

We used dissociated hippocampal neurons transfected with EGFP to visualize spine morphology (Fig. 12). Quantification of spine density along the proximal dendrites and classification of spine phenotype, namely mushroom spines with a head diameter $>0.6 \mu \mathrm{m}$ and thin spines with a head diameter $<0.6 \mu \mathrm{m}$, revealed that the number of mushroom spines was significantly lower in male than in female neurons under control conditions (Fig. 12C). Letrozole treatment resulted in a decrease in the number of large spines by $32 \%$ in female neurons, while no effect was seen in male neurons. Thin spines were reduced by $27 \%$ in female neurons and by $15 \%$ in male neurons (Fig. 12D). As a result, letrozole treatment of dissociated female neurons decreased spine number by $30 \%$ and affected both thin and mushroom spines. In male neurons, spine number was reduced by $9 \%$ and only thin spines were affected (Fig. 12E). The reduction in mature spines in female neurons, together with the inability to induce LTP in females and in female hippocampal slice cultures after $7 \mathrm{~d}$ of letrozole treatment, is in agreement with the findings of Matsuzaki et al. (2004) showing that LTP causes spines to develop a round configuration. Moreover, spine synapse loss in females appears to preferentially concern synapses with large spines rather than thin spines. In males, the number of spine synapses and the number of mature spines were unchanged.

\section{Discussion}

The results of the present study show for the first time that systemic inhibition of aromatase activity significantly impairs LTP and transiently induces dephosphorylation of cofilin in the hippocampus of female, ovariectomized, and male mice. The effects were remarkably prominent in female mice, where LTP was finally abolished, followed by ovariectomized animals and males. As a result of LTP impairment, both types of spines and spine synapses are lost in females, while only thin spines are lost in males. The differences seen between males and females, the results from additional in vitro studies, and our finding that ovariectomy does not influence LTP, all point to a role of hippocampus-derived estrogen, rather than to a role of estrogen from peripheral sources, for the LTP and spine synapse stability.

\section{Aromatase inhibition results in dephosphorylation of cofilin and impairment of LTP}

The essential role of the actin cytoskeleton, which is regulated by numerous proteins, in synaptic plasticity has been extensively shown (for review see Tada and Sheng, 2006; Bramham, 2008; Kasai et al., 2010). In response to the inhibition of aromatase, we found impaired LTP together with a transient reduction of phosphorylated cofilin. This holds true for intact female mice, ovariectomized females, and male mice and was confirmed by findings in hippocampal slice cultures of females. These results are consistent with the findings by Fukazawa et al. (2003) showing that LTP is accompanied by enhanced F-actin and phosphorylation of cofilin.

In stable spines, disassembly of $\mathrm{F}$-actin is prevented as soon as cofilin, an actin-associated protein, becomes phosphorylated. Cofilin is itself regulated; it becomes inactive when phosphorylated by LIM-1 kinase, thus allowing polymerization to proceed (Bramham, 2008). Kramár at al. (2009) showed that application of estradiol to acute male hippocampal slices increases LTP and phosphorylation of cofilin via activation of LIM-1 kinase. We 

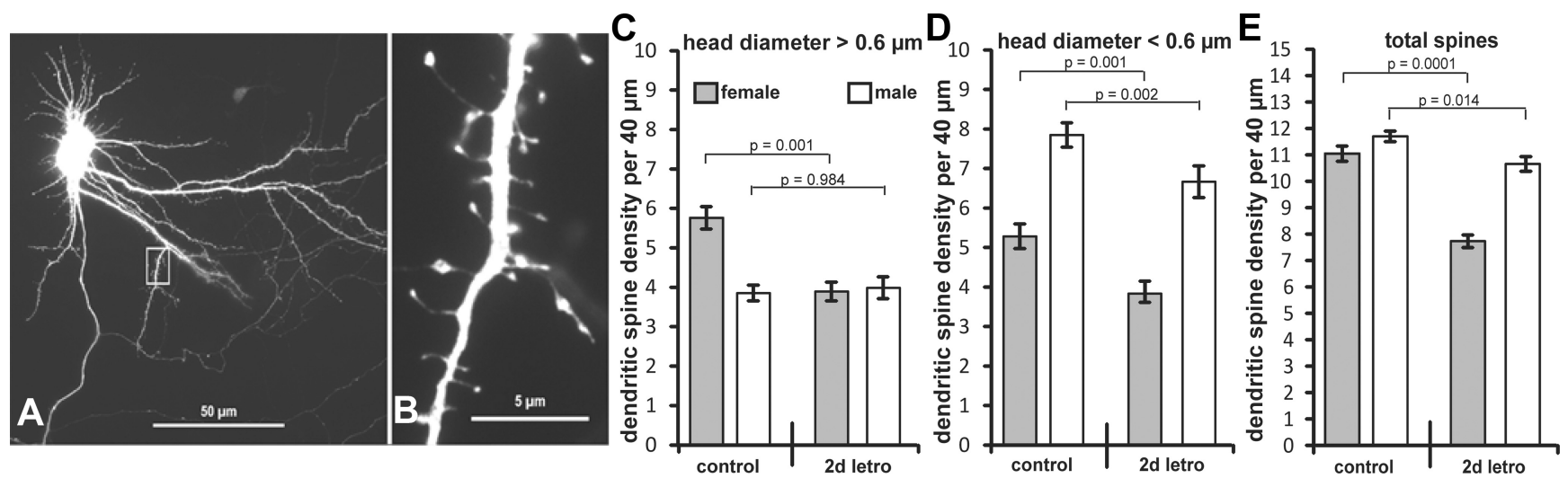

Figure 12. Spine density along dendrites of EGFP transfected neurons. $\boldsymbol{A}$, Spine densities on dendrites of single dissociated neurons transfected with EGFP. $\boldsymbol{B}$, Enlargement of the framed dendrite section in $\boldsymbol{A}$. C $-\boldsymbol{E}$, Determination of spine types (mature spines with a head diameter of $>0.6 \mu \mathrm{m}$ and thin spines with a head diameter $<0.6 \mu \mathrm{m}$ ) revealed more mature spines on female neurons than on male neurons. After $2 \mathrm{~d}$ of treatment of the cultures with letrozole (Letro), loss of mature and thin spines by 32 and $27 \%$ respectively was observed on female dendrites, while on male neurons solely a reduction of thin spines by $15 \%$ was seen. (means \pm SEM; Mature spines: Control, $5.76 \pm 0.28$, Letro, $3.89 \pm 0.24$ (female); Control: $3.85 \pm 0.20$, Letro, $3.99 \pm 0.27$ (male). Thin spines: Control, $5.28 \pm 0.31$, Letro, $3.84 \pm 0.23$ (female); Control, $7.85 \pm 0.31$, Letro, $6.67 \pm 0.40$ (male). Total spines: Control, $11.04 \pm 0.52$, Letro, $7.73 \pm 0.41$ (female); Control, $11.70 \pm 0.39$, Letro, $10.65 \pm 0.56$ (male); $n=3$ independent cultures; a total of 30 neurons in each group were used).

consistently found that inhibition of local estradiol synthesis results in dephosphorylation of cofilin and impairment of LTP.

Our data, however, suggest that LTP and cofilin phosphorylation may be independent phenomena. The downregulation of p-cofilin by letrozole was found in hippocampal slice cultures, independent of theta-burst stimulation. In addition, theta-burst stimulation-induced phosphorylation of cofilin, as shown by Fukazawa et al. (2003), and which was confirmed by our findings, was unable to rescue letrozole-induced dephosphorylation of cofilin. Finally, the time dependency of letrozole effects differed with respect to LTP and p-cofilin. After $7 \mathrm{~d}$ of letrozole treatment of male and female mice, downregulation of p-cofilin immunoreactivity was abolished, while LTP was still impaired.

\section{Spine synapse loss in females but not in males}

We have previously demonstrated that inhibition of aromatase activity in hippocampal neurons induces spine and spine synapse loss in vitro as well as in situ in females (Kretz et al., 2004; Zhou et al., 2010). In this study, we show that aromatase activity in hippocampal neurons is required for theta-burst stimulationinduced LTP and regulates phosphorylation of cofilin. With respect to our results on the time dependency of the effects after aromatase inhibition, it clearly turned out that LTP impairment and dephosphorylation of cofilin precedes spine and spine synapse loss. In all groups of animals, LTP and immunoreactivity of p-cofilin was significantly reduced after $1 \mathrm{~d}$. Spine and spine synapse loss, however, was not seen before $2 \mathrm{~d}$ had passed. This finding suggests that spine synapse loss results from impaired LTP and dephosphorylation of cofilin. This hypothesis is supported by the study of Yuste and Bonhoeffer (2001) showing that LTP induces the formation of spines. Regarding phosphorylation of cofilin, we know that LIM-1 kinase phosphorylates cofilin, which means that our hypothesis is supported by the finding that LIM-1 kinase knock-out mice produce spines that lack actin and have small synapses (Meng et al. 2002). Regarding the time course of p-cofilin reduction, however, which, unlike the time course of LTP impairment, does not correlate with spine and spine synapse reduction, we presume that LTP impairment underlies synapse loss after inhibition of aromatase in mice.

Spine synapse loss in females, but not in males, and differences in the degree of LTP impairment (LTP was abolished in females but was only impaired by $20 \%$ in males after $7 \mathrm{~d}$ of treatment) also suggest that LTP impairment rather than cofilin activity is causal in final spine synapse loss. In males, reduction of p-cofilin immunoreactivity is similar to the reduction in females. Males and females, however, highly differ in the degree of LTP impairment: reduction by $20 \%$ in male mice versus abolishment of LTP in female mice. LTP reduction by $20 \%$ in males may induce loss of thin spines in dissociated neurons, but may not be sufficient to induce spine synapse loss. In this context, it is useful to consider that testosterone induces spine formation in males but not females (Leranth et al., 2004). Concentrations of testosterone in tissue as well as in serum should increase after letrozole treatment, since testosterone is the direct substrate of aromatase. Along these lines, our data show that the density of mature spines, considered to be memory spines, is far lower in male dissociated neurons than in female dissociated neurons. Mature spines are considerably reduced in response to letrozole in female neurons but are unaffected in male neurons. Moreover, spine synapse density is generally lower in male than in female hippocampi, consistent with lower estradiol concentrations in hippocampal tissue of males than of females (Fester et al., 2011). In sum, differences between genders in the regulation of synaptic plasticity in response to sexual steroids show that inhibition of aromatase specifically affects synaptic plasticity in females but not in males.

To test whether estradiol of ovarian origin participated in estrogen-induced synaptic plasticity in the hippocampus, we removed the ovaries, which is a common experimental approach. Strikingly, ovariectomy alone, which substantially reduces serum concentrations in females, had no effect on LTP, despite the loss of spines, as originally shown by Gould et al. (1990). This result confirms findings by Day and Good (2005). Our data clearly show that impaired LTP has nothing to do with spine and spine synapse loss after ovariectomy. Most strikingly, it appears that ovariectomy even protects against abolishment of LTP after long-lasting letrozole treatment. LTP was impaired by $50 \%$ in ovariectomized animals but was abolished in intact females after $7 \mathrm{~d}$ of letrozole treatment. In this context, it has to be noted that the removal of ovaries may take away relevant substances other than merely estradiol. In contrast, our findings in female hippocampal slice cultures, thus in the absence of any other source of 
estradiol, are highly consistent with our data from female mice. Although we cannot completely rule out any other influence of nonhippocampal sources of estradiol, such as the adrenals, our data in hippocampal slice cultures and the finding that ovariectomy does not influence LTP give ample support to the idea that hippocampus-derived estradiol is a main player in estrogeninduced synaptic plasticity in the hippocampus.

\section{Aromatase inhibitors in the therapy of breast cancer}

Aromatase inhibitors, such as letrozole, are commonly used in the therapy of postmenopausal women who suffer from hormonedependent breast cancer (Geisler et al., 2002; Puddefoot et al., 2002). Clinical pilot studies have demonstrated that aromatase-inhibitors affect cognition and memory deficits in women treated with these inhibitors (Shilling et al., 2001, 2005; Dowsett et al., 2005). Letrozole is easily transported across the blood-brain barrier after systemic application and exerts an inhibitory influence on hippocampal estrogen synthesis, as it does in other regions of the body (Zhou et al., 2010). Impaired LTP, as an electrophysiological parameter of memory, together with spine synapse loss (Lamprecht and LeDoux, 2004), after estradiol depletion, could be the underlying mechanisms for memory deficits in women treated with aromatase inhibitors for therapeutical reasons. The results point to the necessity of further clinical studies on the effects of letrozole on cognitive deficits in women.

\section{References}

Abramoff MD, Magalhaes PJ, Ram SJ (2004) Processing with ImageJ. Biophotonics International 11:36-42.

Behnisch T, Matsushita S, Knöpfel T (2004) Imaging of gene expression during long-term potentiation. Neuroreport 15:2039-2043.

Braendgaard H, Gundersen HJ (1986) The impact of recent stereological advances on quantitative studies of the nervous system. J Neurosci Methods 18:39-78.

Bramham CR (2008) Local protein synthesis, actin dynamics, and LTP consolidation. Curr Opin Neurobiol 18:524-531.

Bramham CR, Worley PF, Moore MJ, Guzowski JF (2008) The immediate early gene Arc/Arg3.1: regulation, mechanisms, and function. J Neurosci 28:11760-11767.

Bukalo O, Fentrop N, Lee AY, Salmen B, Law JW, Wotjak CT, Schweizer M, Dityatev A, Schachner M (2004) Conditional ablation of the neural cell adhesion molecule reduces precision of spatial learning, long-term potentiation, and depression in the CA1 subfield of mouse hippocampus. J Neurosci 24:1565-1577.

Córdoba Montoya DA, Carrer HF (1997) Estrogen facilitates induction of long term potentiation in the hippocampus of awake rats. Brain Res 778:430-438

Day M, Good M (2005) Ovariectomy-induced disruption of long-term synaptic depression in the hippocampal CA1 region in vivo is attenuated with chronic estrogen replacement. Neurobiol Learn Mem 83:13-21.

Dowsett M, Haynes BP (2003) Hormonal effects of aromatase inhibitors: focus on premenopausal effects and interaction with tamoxifen. J Steroid Biochem Mol Biol 86:255-263.

Dowsett M, Folkerd E, Doody D, Haynes B (2005) The biology of steroid hormones and endocrine treatment of breast cancer. Breast 14:452-457.

Fester L, Ribeiro-Gouveia V, Prange-Kiel J, von Schassen C, Böttner M, Jarry H, Rune GM (2006) Proliferation and apoptosis of hippocampal granule cells require local oestrogen synthesis. J Neurochem 97:1136-1144.

Fester L, Zhou L, Voets C, Ossig C, Disteldorf E, Bläute F, Prange-Kiel J, Dudzinski D, Jarry H, Rune GM (2009) The opposing roles of estradiol on synaptic protein expression in hippocampal cultures. Psychoneuroendocrinology 34 [Suppl 1]:S123-S129.

Fester L, Prange-Kiel J, Zhou L, von Blittersdorf B, Böhm J, Jarry H, Schumacher M, Rune GM (2011) Estrogen-regulated synaptogenesis: sexual dimorphism in vivo but not in vitro. J Steroid Biochem Mol Biol. Advance online publication. Retrieved November 25, 2011. PMID: 22138012.

Fiala JC, Spacek J, Harris KM (2002) Dendritic spine pathology: cause or consequence of neurological disorders? Brain Res Brain Res Rev 39:29-54.
Foy MR, Xu J, Xie X, Brinton RD, Thompson RF, Berger TW (1999) 17betaestradiol enhances NMDA receptor-mediated EPSPs and long-term potentiation. J Neurophysiol 81:925-929.

Fukazawa Y, Saitoh Y, Ozawa F, Ohta Y, Mizuno K, Inokuchi K (2003) Hippocampal LTP is accompanied by enhanced F-actin content within the dendritic spine that is essential for late LTP maintenance in vivo. Neuron 38:447-460.

Geisler J, Haynes B, Anker G, Dowsett M, Lønning PE (2002) Influence of letrozole and anastrozole on total body aromatization and plasma estrogen levels in postmenopausal breast cancer patients evaluated in a randomized, cross-over study. J Clin Oncol 20:751-757.

Gould E, Woolley CS, Frankfurt M, McEwen BS (1990) Gonadal steroids regulate dendritic spine density in hippocampal pyramidal cells in adulthood. J Neurosci 10:1286-1291.

Ito K, Skinkle KL, Hicks TP (1999) Age-dependent, steroid-specific effects of oestrogen on long-term potentiation in rat hippocampal slices. J Physiol 515 [Pt 1]:209-220.

Kasai H, Fukuda M, Watanabe S, Hayashi-Takagi A, Noguchi J (2010) Structural dynamics of dendritic spines in memory and cognition. Trends Neurosci 33:121-129.

Kramár EA, Chen LY, Brandon NJ, Rex CS, Liu F, Gall CM, Lynch G (2009) Cytoskeletal changes underlie estrogen's acute effects on synaptic transmission and plasticity. J Neurosci 29:12982-12993.

Kretz O, Fester L, Wehrenberg U, Zhou L, Brauckmann S, Zhao S, PrangeKiel J, Naumann T, Jarry H, Frotscher M, Rune GM (2004) Hippocampal synapses depend on hippocampal estrogen synthesis. J Neurosci 24:5913-5921.

Lamprecht R, LeDoux J (2004) Structural plasticity and memory. Nat Rev Neurosci 5:45-54.

Leranth C, Hajszan T, MacLusky NJ (2004) Androgens increase spine synapse density in the CA1 hippocampal subfield of ovariectomized female rats. J Neurosci 24:495-499.

Lynch G (2004) AMPA receptor modulators as cognitive enhancers. Curr Opin Pharmacol 4:4-11.

Malenka RC, Nicoll RA (1999) Neuroscience-long-term potentiation-a decade of progress? Science 285:1870-1874.

Matsuzaki S, Canis M, Vaurs-Barrière C, Pouly JL, Boespflug-Tanguy $\mathrm{O}$, Penault-Llorca F, Dechelotte P, Dastugue B, Okamura K, Mage G (2004) DNA microarray analysis of gene expression profiles in deep endometriosis using laser capture microdissection. Mol Hum Reprod 10:719-728.

Matus A (2000) Actin-based plasticity in dendritic spines. Science 290:754-758.

Mellentin C, Møller M, Jahnsen H (2006) Properties of long-term synaptic plasticity and metaplasticity in organotypic slice cultures of rat hippocampus. Exp Brain Res 170:522-531.

Mendez P, Garcia-Segura LM, Muller D (2011) Estradiol promotes spine growth and synapse formation without affecting preestablished networks. Hippocampus 21:1263-1267.

Meng Y, Zhang Y, Tregoubov V, Janus C, Cruz L, Jackson M, Lu WY, MacDonald JF, Wang JY, Falls DL, Jia Z (2002) Abnormal spine morphology and enhanced LTP in LIMK-1 knock-out mice. Neuron 35:121-133.

Mukai H, Tsurugizawa T, Murakami G, Kominami S, Ishii H, Ogiue-Ikeda M, Takata N, Tanabe N, Furukawa A, Hojo Y, Ooishi Y, Morrison JH, Janssen WG, Rose JA, Chambon P, Kato S, Izumi S, Yamazaki T, Kimoto T, Kawato S (2007) Rapid modulation of long-term depression and spinogenesis via synaptic estrogen receptors in hippocampal principal neurons. J Neurochem 100:950-967.

Muller D, Wang C, Skibo G, Toni N, Cremer H, Calaora V, Rougon G, Kiss JZ (1996) PSA-NCAM is required for activity-induced synaptic plasticity. Neuron 17:413-422.

Murphy DD, Segal M (1996) Regulation of dendritic spine density in cultured rat hippocampal neurons by steroid hormones. J Neurosci 16:4059-4068

Podlogar M, Dietrich D (2006) Firing pattern of rat hippocampal neurons: a perforated patch clamp study. Brain Res 1085:95-101.

Prange-Kiel J, Wehrenberg U, Jarry H, Rune GM (2003) Para/autocrine regulation of estrogen receptors in hippocampal neurons. Hippocampus 13:226-234.

Prange-Kiel J, Rune GM, Leranth C (2004) Median raphe mediates estrogenic effects to the hippocampus in female rats. Eur J Neurosci 19:309-317.

Prange-Kiel J, Fester L, Zhou L, Lauke H, Carrétero J, Rune GM (2006) 
Inhibition of hippocampal estrogen synthesis causes region-specific downregulation of synaptic protein expression in hippocampal neurons. Hippocampus 16:464-471.

Puddefoot JR, Barker S, Glover HR, Malouitre SD, Vinson GP (2002) Noncompetitive steroid inhibition of oestrogen receptor functions. Int J Cancer 101:17-22.

Rune GM, Frotscher M (2005) Neurosteroid synthesis in the hippocampus: role in synaptic plasticity. Neuroscience 136:833-842.

Rune GM, Lohse C, Prange-Kiel J, Fester L, Frotscher M (2006) Synaptic plasticity in the hippocampus: effects of estrogen from the gonads or hippocampus? Neurochem Res 31:145-155.

Shi Y, Ethell IM (2006) Integrins control dendritic spine plasticity in hippocampal neurons through NMDA receptor and $\mathrm{Ca}^{2+} /$ calmodulindependent protein kinase II-mediated actin reorganization. J Neurosci 26:1813-1822.

Shi Y, Pontrello CG, DeFea KA, Reichardt LF, Ethell IM (2009) Focal adhesion kinase acts downstream of EphB receptors to maintain mature dendritic spines by regulating cofilin activity. J Neurosci 29:8129-8142.

Shilling V, Jenkins V, Fallowfield L, Howell A (2001) The effects of oestrogens and anti-oestrogens on cognition. Breast 10:484-491.

Shilling V, Jenkins V, Morris R, Deutsch G, Bloomfield D (2005) The effects of adjuvant chemotherapy on cognition in women with breast cancerpreliminary results of an observational longitudinal study. Breast 14:142-150.

Smith CC, McMahon LL (2005) Estrogen-induced increase in the magnitude of long-term potentiation occurs only when the ratio of NMDA transmission to AMPA transmission is increased. J Neurosci 25:77807791.

Smith CC, McMahon LL (2006) Estradiol-induced increase in the magnitude of long-term potentiation is prevented by blocking NR2Bcontaining receptors. J Neurosci 26:8517-8522.

Snyder MA, Cooke BM, Woolley CS (2011) Estradiol potentiation of
NR2B-dependent EPSCs is not due to changes in NR2B protein expression or phosphorylation. Hippocampus 21:398-408.

Spencer JL, Waters EM, Milner TA, McEwen BS (2008) Estrous cycle regulates activation of hippocampal Akt, LIM kinase, and neurotrophin receptors in C57BL/6 mice. Neuroscience 155:1106-1119.

Sterio DC (1984) The unbiased estimation of number and sizes of arbitrary particles using the disector. J Microsc 134:127-136.

Stoppini L, Buchs PA, Muller D (1991) A simple method for organotypic cultures of nervous tissue. J Neurosci Methods 37:173-182.

Tada T, Sheng M (2006) Molecular mechanisms of dendritic spine morphogenesis. Curr Opin Neurobiol 16:95-101.

Walf AA, Rhodes ME, Frye CA (2006) Ovarian steroids enhance object recognition in naturally cycling and ovariectomized, hormone-primed rats. Neurobiol Learn Mem 86:35-46.

Warren SG, Humphreys AG, Juraska JM, Greenough WT (1995) LTP varies across the estrous cycle: Enhanced synaptic plasticity in proestrus rats. Brain Res 703:26-30.

Wehrenberg U, Prange-Kiel J, Rune GM (2001) Steroidogenic factor-1 expression in marmoset and rat hippocampus: colocalization with StAR and aromatase. J Neurochem 76:1879-1886.

Yuste R, Bonhoeffer T (2001) Morphological changes in dendritic spines associated with long-term synaptic plasticity. Annu Rev Neurosci 24:1071-1089.

Yuste R, Bonhoeffer T (2004) Genesis of dendritic spines: insights from ultrastructural and imaging studies. Nat Rev Neurosci 5:24-34.

Zhou L, Lehan N, Wehrenberg U, Disteldorf E, von Lossow R, Mares U, Jarry H, Rune GM (2007) Neuroprotection by estradiol: a role of aromatase against spine synapse loss after blockade of GABA(A) receptors. Exp Neurol 203:72-81.

Zhou L, Fester L, von Blittersdorff B, Hassu B, Nogens H, Prange-Kiel J, Jarry H, Wegscheider K, Rune GM (2010) Aromatase inhibitors induce spine synapse loss in the hippocampus of ovariectomized mice. Endocrinology 151:1153-1160. 